\title{
Macroscopic-microscopic model of nuclear potential energy
}

\section{Implementation in the CONRAD code}

\author{
Pierre Tamagno ${ }^{1, a}$, Olivier Bouland ${ }^{1}$, Olivier Serot $^{1}$, and Peter Moller ${ }^{2}$ \\ 1 CEA, DEN, DER, SPRC, Cadarache, 13108 Saint-Paul-lez-Durance, France \\ 2 Los Alamos National Laboratory, Theoretical Division, Los Alamos, NM 87545, USA
}

\begin{abstract}
To improve the evaluation of nuclear observables, refined models are to be used more and more as underlying analysis tools. Fission is a complex process and is the less accurately described with current models. Standard evaluation models rely on the Hill-Wheeler formalism for the fission transmission coefficient, which in turns is based on phenomenological parameters "reflecting" the fission barrier heights and widths. To reduce the weight of phenomenology in the evaluation process, nuclear structure models are expected to embed more and more microscopic descriptions. As models are rarely exact, evaluators are often compelled to "tune" model parameters so that observables can be properly reproduced. Related computation time can thus be a major hindrance to the use of advanced models in evaluation as final adjustments are expected to remain necessary. For this reason, a macroscopic-microscopic model has been selected to replace the current phenomenological description of fission barriers. The Finite-Range Liquid-Drop Model (FRLDM) has been implemented in the CONRAD evaluation code and its present implementation shows remarkable consistency with experimental and published benchmark data. The CONRAD code can be used to provide expectation values but also related uncertainties and covariance data. Sensitivity of FRLDM parameters and the correlation matrix between these parameters have been obtained so that further uncertainty propagation on barrier heights can be carried out in the near future.
\end{abstract}

\section{Introduction and motivations}

Most of phenomenological models to evaluate fission cross sections rely on the Hill-Wheeler expression for fission transmission coefficient [1]. For a compound nucleus with excitation energy $E^{*}$ it reads

$$
T_{\mathrm{f}}^{\mathrm{HW}}\left(E^{*}\right)=\left[1+\exp \left(2 \pi \frac{V_{\mathrm{f}}-E^{*}}{\hbar \omega}\right)\right]^{-1} .
$$

In this model, the phenomenological parameters $V_{\mathrm{f}}$ and $\hbar \omega$ are respectively associated to the fundamental fission barrier height and curvature. Yet in practice these parameters are adjusted so that evaluator can properly reproduce required observables - usually the fission cross section.

The use of such phenomenological parameters in the evaluation process limits the possibility for evaluator to predict observables for which no experimental data are available. The general trend in the evaluation process thus consists in involving more fundamental models that could be used to provide such intermediate parameters.

The macroscopic-microscopic Finite-Range LiquidDrop Model [2] (FRLDM) is a good candidate to achieve this goal as demonstrated by correct nucleus mass predictions, i.e. the binding energy of atomic nuclei. As this model can be used to calculate the nucleus energy as it deforms, it can be also used to obtain an estimation of fission barrier heights $V_{\mathrm{f}}$.

a e-mail: pierre.tamagno@cea.fr
Best estimate values are not the only required data to perform a proper evaluation. Indeed evaluators must also propagate uncertainties on model parameters to the evaluated quantities. Therefore we have implemented the FRLDM in the CONRAD evaluation code [3], as this was designed to perform data assimilation and uncertainty propagation. The proper method to propagate uncertainties on fission barrier heights would consist in adjusting model parameters for masses to obtain both central values and uncertainties on parameters. Yet as the experimental precision on masses is very high, it is expected that such an approach yields nonphysically small uncertainty on parameters. This is an example of model-defect, which is a wide issue in evaluation. Therefore we restrained the present study to sensitivities and correlation matrix on model parameters. As all model parameters have been considered, including microscopic parameters, semi-analytical sensitivities have been derived and implemented in the code. In this paper we summarize the model parameters and provide semianalytical sensitivities expressions. Finally, sensitivity results as well as a correlation matrix are presented.

\section{Macroscopic-microscopic model}

The FRLDM is comprehensively described in Ref. [2], so we recall here only the main lines to highlight the fundamental model parameters. For undefined quantities, the reader should refer to Ref. [2]. 


\subsection{Macroscopic contribution}

In the FRLDM, atomic mass for a nucleus with a shape $\vec{q}$ contains a macroscopic term given by Eq. (2), in which the 13 parameters are highlighted in red color.

$$
\begin{aligned}
& E_{\text {macro }}^{\mathrm{FRLDM}}(Z, A, \vec{q})=M_{\mathrm{H}} Z+M_{\mathrm{n}} N-a_{\mathrm{V}}\left(1-\kappa_{\mathrm{V}} I^{2}\right) A \\
& +a_{\mathrm{S}}\left(1-\kappa_{\mathrm{S}} I^{2}\right) B_{1}\left(a, r_{0}, \vec{q}\right) A^{2 / 3} \\
& +f\left(k_{\mathrm{F}} r_{\mathrm{p}}\right) \frac{Z^{2}}{A}-c_{\mathrm{a}}(N-Z) \\
& +c_{1} \frac{Z^{2}}{A^{1 / 3}} B_{3}\left(a_{\mathrm{den}}, r_{0}, \vec{q}\right)-c_{4} \frac{Z^{4 / 3}}{A^{1 / 3}} \\
& +W\left(|I|+\frac{1}{A} \delta_{Z N} \delta_{\text {Zodd }}\right) \\
& +a_{0}+ \begin{cases}\bar{\Delta}_{\mathrm{p}}+\bar{\Delta}_{\mathrm{n}}-\delta_{\mathrm{np}}, & Z \& N \text { odd } \\
\bar{\Delta}_{\mathrm{p}}, & Z \text { odd \& } N \text { even } \\
\bar{\Delta}_{\mathrm{n}}, & Z \text { even \& } N \text { odd } \\
0, & Z \text { \& } N \text { even }\end{cases}
\end{aligned}
$$

where $c_{1}, c_{4}$ and $k_{\mathrm{F}}$ depend only on $r_{0}$ according to

$$
c_{1}=\frac{3}{5} \frac{e^{2}}{r_{0}}, c_{4}=\frac{5}{4} c_{1}\left(\frac{3}{2 \pi}\right)^{2 / 3}, k_{\mathrm{F}}=\frac{1}{r_{0}}\left(\frac{9 \pi Z}{4 A}\right)^{1 / 3}
$$

and where the macroscopic pairing energy is given by

$$
\bar{\Delta}_{\mathrm{n}}=\frac{r_{\mathrm{mac}} B_{\mathrm{S}}(\vec{q})}{N^{1 / 3}}, \quad \bar{\Delta}_{\mathrm{p}}=\frac{r_{\mathrm{mac}} B_{\mathrm{S}}(\vec{q})}{Z^{1 / 3}}, \quad \delta_{\mathrm{np}}=\frac{h A^{-2 / 3}}{B_{\mathrm{S}}(\vec{q})} .
$$

\subsection{Microscopic contribution}

The microscopic contribution to the atomic mass consists of a shell and a pairing corrections. Both of these are calculated from the single-particles energies obtained from an independent-particle model. The phenomenological mean potentials of the model are given by

$$
\begin{gathered}
\hat{V}_{\mathrm{n}}\left(\vec{r}_{1}\right)=-\frac{V_{\mathrm{n} / \mathrm{p}}}{4 \pi a_{\mathrm{pot}}{ }^{3}} \int_{V} \frac{\mathrm{e}^{\left\|\vec{r}_{1}-\vec{r}_{2}\right\| / a_{\mathrm{pot}}}}{\left\|\vec{r}_{1}-\vec{r}_{2}\right\| / a_{\mathrm{pot}}} \mathrm{d}^{3} \vec{r}_{2}, \\
\hat{V}_{\mathrm{C}}\left(\vec{r}_{1}\right)=\frac{Z e^{2}}{\frac{4}{3} \pi R_{\mathrm{pot}}^{3}} \int_{V} \frac{\mathrm{d}^{3} \vec{r}_{2}}{\left\|\vec{r}_{1}-\vec{r}_{2}\right\|}, \\
\hat{V}_{\text {s.o. }}=-\lambda_{\mathrm{n} / \mathrm{p}}\left(\frac{\hbar}{2 m c}\right)^{2} \overline{\bar{\sigma}} \cdot \vec{\nabla} V_{\mathrm{N}} \times \frac{\vec{p}}{\hbar},
\end{gathered}
$$

where the potential well depths $V_{\mathrm{n} / \mathrm{p}}$ and spin-orbit coupling strengths $\lambda_{\mathrm{n} / \mathrm{p}}$ are given by

$$
\begin{array}{ll}
V_{\mathrm{n}}=V_{\mathrm{s}}-\bar{\delta} V_{\mathrm{a}}, & V_{\mathrm{p}}=V_{\mathrm{s}}+\bar{\delta} V_{\mathrm{a}}, \\
\lambda_{\mathrm{n}}=k_{\mathrm{n}} A+l_{\mathrm{n}}, & \lambda_{\mathrm{p}}=k_{\mathrm{p}} A+l_{\mathrm{p}} .
\end{array}
$$

The surface containing the volume $V$ in Eq. (8) corresponds to an equivalent sphere of radius $R_{\text {pot }}$ given by

$$
R_{\mathrm{pot}}=A_{\mathrm{den}}+R_{\mathrm{den}}-\frac{B_{\mathrm{den}}}{R_{\mathrm{den}}}, \quad R_{\mathrm{den}}=r_{0} A^{1 / 3}(1+\bar{\epsilon}) .
$$

Finally, the two droplet-model quantities $\bar{\delta}$ and $\bar{\epsilon}$ are given by

$$
\begin{gathered}
\bar{\delta}=\left(I+\frac{3}{8} \frac{c_{1}}{Q} \frac{Z^{2}}{A^{5 / 3}}\right)\left(1+\frac{9}{4} \frac{J}{Q} A^{-1 / 3}\right)^{-1}, \\
\bar{\epsilon}=\frac{1}{K}\left(-\frac{2 a_{2}}{A^{1 / 3}}+L \bar{\delta}^{2}+c_{1} \frac{Z^{2}}{A^{4 / 3}}\right) .
\end{gathered}
$$

The microscopic term thus involves 15 parameters. From the single-particle energies calculated using the mean potential of Eq. (8), shell and pairing corrections are calculated using the Strutinsky and Lipkin-Nogami formalisms [2], which involve two additional parameters, the Strutinsky range coefficient $C_{\mathrm{S}}$ and the LipkinNogami effective-interaction pairing-gap constant $r_{\text {mic }}$. Parameter $r_{\text {mac }}$ has no impact on calculated masses as it is strictly compensated in the pairing correction term. Finally, the zero-vibration energy involves a last parameter $R$.

The FRLDM model has been implemented in CONRAD from scratch. Necessary verification against experimental masses and published data showed satisfactory consistency. This verification is illustrated in Fig. 1. The upper part of the figure presents comparison with experimental masses [2] whereas the lower part shows the differences with the original implementation by Möller et al. [2]. We chose to work with rather old evaluated masses so that we can ensure consistency between model implementations. For the present purpose, which is to provide a correlation matrix between model parameters, these consistencies are satisfactory. The standard deviation with experimental data is less than $0.769 \mathrm{MeV}$, which is only $15 \mathrm{keV}$ different from the original implementation.

\section{Parameter sensitivity}

\subsection{Macroscopic model parameters}

Data analysis requires to know how model parameters impact theoretical values. CONRAD needs then to compute sensitivities i.e. derivatives of theoretical values $M^{\text {th }}$ with respect of model parameters. The code offers two possibilities; it can perform numerical derivatives using the model as a black-box (readily available for all new models), or one can bypass this calculation by implementing analytical derivatives if available. In the present case, we used this latter solution as singleparticle energies computation takes significant amount of time. A first simplification is to avoid single-particle energies computations whenever macroscopic parameters $p_{\text {macro }}$ are varied, $\partial M^{\text {th }} / \partial p_{\text {macro }}=\partial E_{\text {macro }}^{\mathrm{FRLD}} / \partial p_{\text {macro }}$. This can be further improved as most macroscopic-parameters derivatives are straightforward, as long as no shape 

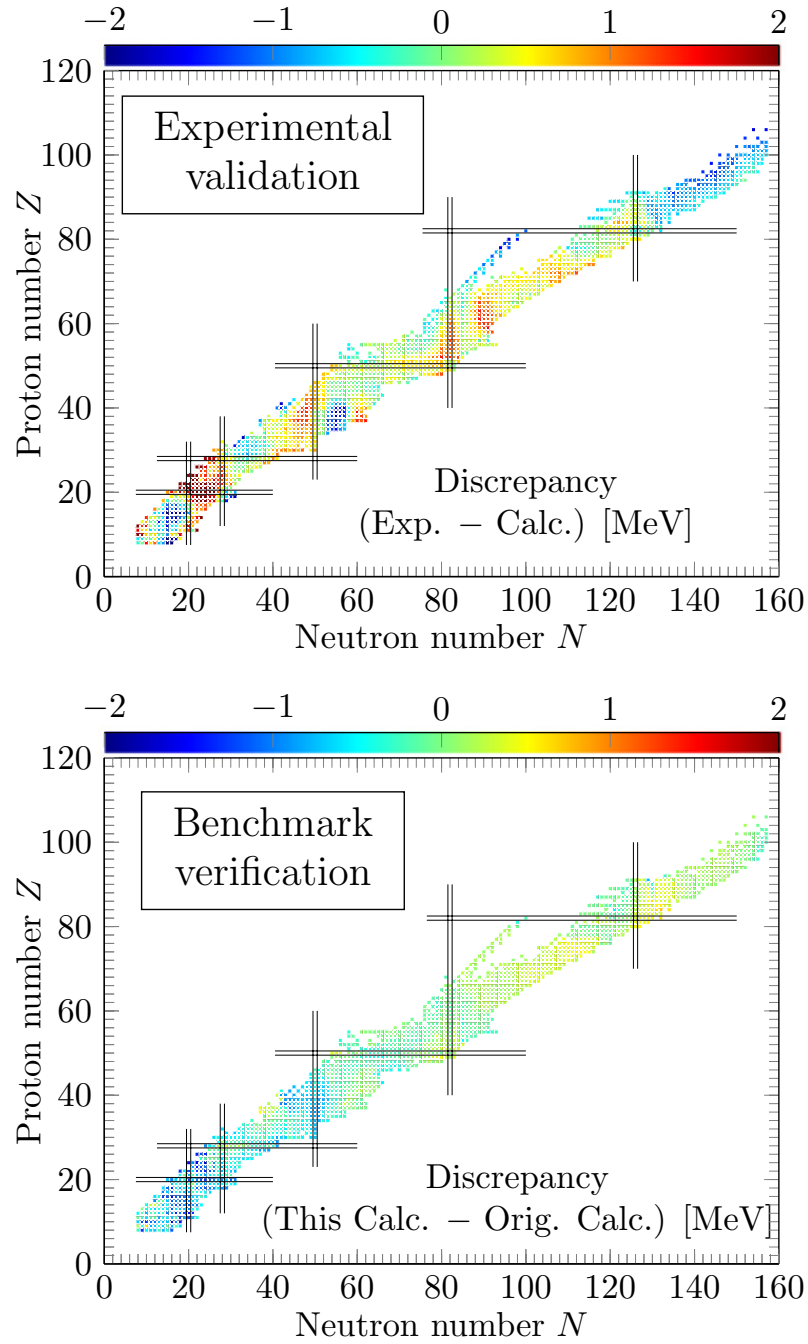

Figure 1. Comparison between CONRAD theoretical masses and experimental masses (upper part) and with previously published values (lower part).

variation is assumed. Namely:

$$
\begin{array}{rlrl}
\frac{\partial M^{\text {th }}}{\partial a_{\mathrm{V}}} & =-\left(1-\kappa_{\mathrm{V}} I^{2}\right) A, & \frac{\partial M^{\text {th }}}{\partial \kappa_{\mathrm{V}}} & =a_{\mathrm{V}} I^{2} A, \\
\frac{\partial M^{\text {th }}}{\partial a_{\mathrm{S}}} & =\left(1-\kappa_{\mathrm{S}} I^{2}\right) B_{1} A^{2 / 3}, & \frac{\partial M^{\text {th }}}{\partial \kappa_{\mathrm{S}}} & =-a_{\mathrm{S}} I^{2} B_{1} A^{2 / 3}, \\
\frac{\partial M^{\text {th }}}{\partial W} & =|I|+\frac{1}{A} \delta_{Z N} \delta_{Z_{\text {odd }}}, & \frac{\partial M^{\text {th }}}{\partial c_{a}} & =Z-N, \\
\frac{\partial M^{\text {th }}}{\partial r_{\text {mac }}} & =B_{\mathrm{S}}\left(\frac{\delta_{\text {Zodd }}}{Z^{1 / 3}}+\frac{\delta_{N \text { odd }}}{N^{1 / 3}}\right), \frac{\partial M^{\text {th }}}{\partial a_{0}}=1, \\
\frac{\partial M^{\text {th }}}{\partial h} & =-\frac{\delta_{\text {Zodd }} \delta_{N \text { odd }}}{A^{2 / 3} B_{\mathrm{S}}}, & \frac{\partial M^{\text {th }}}{\partial a_{\mathrm{el}}} & =-Z^{2.39} .
\end{array}
$$

For derivatives with respect of $a$ and $a_{\mathrm{den}}$, we perform numerical derivatives for the $B_{1}$ and $B_{3}$ terms respectively, and we use

$$
\frac{\partial M^{\text {th }}}{\partial a}=a_{\mathrm{S}}\left(1-\kappa_{\mathrm{S}} I^{2}\right) A^{2 / 3} \frac{\partial B_{1}}{\partial a}, \frac{\partial M^{\text {th }}}{\partial a_{\mathrm{den}}}=c_{1} \frac{Z^{2}}{A^{1 / 3}} \frac{\partial B_{3}}{\partial a_{\mathrm{den}}},
$$

$$
\frac{\partial M^{\text {th }}}{\partial r_{\mathrm{p}}}=\frac{-e^{2} r_{\mathrm{p}} Z^{2}}{8 r_{0}^{3} A}\left(\frac{145}{24}-\frac{327}{720} k_{\mathrm{F}}^{2} r_{\mathrm{p}}^{2}+\frac{1527}{201600} k_{\mathrm{F}}^{4} r_{\mathrm{p}}^{4}\right)
$$

Finally for $r_{0}$, the corresponding derivative is

$$
\begin{aligned}
\frac{\partial M^{\text {th }}}{\partial r_{0}}= & \frac{Z^{2} r_{\mathrm{p}}^{2} e^{2}}{8 r_{0}^{4} A}\left(\frac{145}{16}-\frac{327}{576} k_{\mathrm{F}}^{2} r_{\mathrm{p}}^{2}+\frac{1527}{172800} k_{\mathrm{F}}^{4} r_{\mathrm{p}}^{4}\right) \\
& +\frac{c_{4} Z^{4 / 3}}{r_{0} A^{1 / 3}}-a_{\mathrm{S}} \frac{a}{r_{0}} \frac{\partial B_{1}}{\partial a}\left(1-\kappa_{\mathrm{S}} I^{2}\right) A^{2 / 3} \\
& -\frac{c_{1} Z^{2}}{A^{1 / 3} r_{0}}\left(a_{\mathrm{den}} \frac{\partial B_{3}}{\partial a_{\mathrm{den}}}+B_{3}\right) .
\end{aligned}
$$

\subsection{Microscopic model parameters}

Microscopic parameters derivatives are more complex to evaluate as analytical derivatives are not available. Yet the number of full single-particle calculations can be reduced. In Sect. 2.2, we can identify 15 parameters involved in the single-particle energies computations: $a_{\mathrm{pot}}, V_{\mathrm{S}}, V_{\mathrm{a}}, k_{\mathrm{n}}$, $k_{\mathrm{p}}, l_{\mathrm{n}}, l_{\mathrm{p}}, A_{\mathrm{den}}, B_{\mathrm{den}}, r_{0}, Q, J, K, a_{2}, L$, which would require at least 16 single-particle energies computations to get all numerical derivatives. This can be reduced by a factor of 3 noticing that single-particles energies only depend on 6 parameters, namely $V_{\mathrm{n}}, V_{\mathrm{p}}, \lambda_{\mathrm{n}}, \lambda_{\mathrm{p}}, R_{\mathrm{pot}}, a_{\text {pot }}$. The derivatives with respect of each the 15 parameters can be deduced from the derivatives of these 6 parameters. We notice here that the microscopic corrections are computed separately for proton and neutron, and will be called $E_{\mathrm{p}}^{\text {mic }}$ and $E_{\mathrm{n}}^{\text {mic }}$ respectively. Therefore we can obtain the impact of varying for instance $V_{\mathrm{n}}$ and $V_{\mathrm{p}}$ separately in a single calculation. In the following, we note $E^{\text {mic }}=E_{\mathrm{p}}^{\text {mic }}+E_{\mathrm{n}}^{\text {mic }}$ and we consider that the following derivatives can be computed numerically:

$$
\frac{\partial E^{\text {mic }}}{\partial R_{\mathrm{pot}}}, \quad \frac{\partial E_{\mathrm{n}}^{\mathrm{mic}}}{\partial \lambda_{\mathrm{n}}}, \quad \frac{\partial E_{\mathrm{p}}^{\mathrm{mic}}}{\partial \lambda_{\mathrm{p}}}, \quad \frac{\partial E_{\mathrm{n}}^{\mathrm{mic}}}{\partial V_{\mathrm{n}}}, \quad \frac{\partial E_{\mathrm{p}}^{\text {mic }}}{\partial V_{\mathrm{p}}} .
$$

From the definitions in Sect. 2.2, one can derive the following relations for the spin-orbit terms:

$$
\frac{\partial E_{\mathrm{n}}^{\mathrm{mic}}}{\partial l_{\mathrm{n}}}=\frac{\partial E_{\mathrm{n}}^{\mathrm{mic}}}{\partial \lambda_{\mathrm{n}}}, \quad \frac{\partial E_{\mathrm{n}}^{\mathrm{mic}}}{\partial k_{\mathrm{n}}}=A \frac{\partial E_{\mathrm{n}}^{\mathrm{mic}}}{\partial \lambda_{\mathrm{n}}}
$$

with a similar expression for the corresponding protonsrelated quantities. For the mean potential well depths:

$$
\frac{\partial E^{\mathrm{mic}}}{\partial V_{\mathrm{S}}}=\frac{\partial E_{\mathrm{n}}^{\mathrm{mic}}}{\partial V_{\mathrm{n}}}+\frac{\partial E_{\mathrm{p}}^{\mathrm{mic}}}{\partial V_{\mathrm{p}}}, \quad \frac{\partial E^{\mathrm{mic}}}{\partial V_{\mathrm{a}}}=\bar{\delta}\left(\frac{\partial E_{\mathrm{p}}^{\mathrm{mic}}}{\partial V_{\mathrm{p}}}-\frac{\partial E_{\mathrm{n}}^{\mathrm{mic}}}{\partial V_{\mathrm{n}}}\right) .
$$

For the spherical equivalent radius:

$$
\frac{\partial E^{\mathrm{mic}}}{\partial A_{\mathrm{den}}}=\frac{\partial E^{\mathrm{mic}}}{\partial R_{\mathrm{pot}}}, \quad \frac{\partial E^{\mathrm{mic}}}{\partial B_{\mathrm{den}}}=-\frac{1}{R_{\mathrm{den}}} \frac{\partial E^{\mathrm{mic}}}{\partial R_{\mathrm{pot}}} .
$$


Then for the droplet quantities:

$$
\begin{aligned}
\frac{\partial E^{\mathrm{mic}}}{\partial L} & =\frac{\partial E^{\mathrm{mic}}}{\partial R_{\mathrm{pot}}}\left(1+\frac{B_{\mathrm{den}}}{R_{\mathrm{den}}^{2}}\right) r_{0} A^{1 / 3} \frac{\bar{\delta}^{2}}{K}, \\
\frac{\partial E^{\mathrm{mic}}}{\partial a_{2}} & =-2 \frac{\partial E^{\mathrm{mic}}}{\partial R_{\mathrm{pot}}}\left(1+\frac{B_{\mathrm{den}}}{R_{\mathrm{den}}^{2}}\right) \frac{r_{0}}{K}, \\
\frac{\partial E^{\mathrm{mic}}}{\partial K} & =-\frac{\partial E^{\mathrm{mic}}}{\partial R_{\mathrm{pot}}}\left(1+\frac{B_{\mathrm{den}}}{R_{\mathrm{den}}^{2}}\right) r_{0} A^{1 / 3} \frac{\bar{\epsilon}}{K}, \\
\frac{\partial E^{\mathrm{mic}}}{\partial J} & =-9 \frac{\partial E^{\mathrm{mic}}}{\partial \bar{\delta}} \bar{\delta}\left(4 Q A^{1 / 3}+9 J\right)^{-1}, \\
\frac{\partial E^{\mathrm{mic}}}{\partial Q} & =\frac{6}{A} \frac{\partial E^{\mathrm{mic}}}{\partial \bar{\delta}} \frac{6 I J A^{4 / 3}-c_{1} Z^{2}}{\left(4 Q A^{1 / 3}+9 J\right)^{2}} .
\end{aligned}
$$

where

$$
\begin{aligned}
\frac{\partial E^{\mathrm{mic}}}{\partial \bar{\delta}}= & \frac{\partial E^{\mathrm{mic}}}{\partial R_{\mathrm{pot}}}\left(1+\frac{B_{\mathrm{den}}}{R_{\mathrm{den}}^{2}}\right) r_{0} A^{1 / 3} \frac{2 L \bar{\delta}}{K} \\
& +V_{a}\left(\frac{\partial E_{\mathrm{p}}^{\mathrm{mic}}}{\partial V_{\mathrm{p}}}-\frac{\partial E_{\mathrm{n}}^{\mathrm{mic}}}{\partial V_{\mathrm{n}}}\right)
\end{aligned}
$$

Finally for $r_{0}$ :

$$
\begin{aligned}
\frac{\partial E^{\mathrm{mic}}}{\partial r_{0}}= & \frac{\partial E^{\mathrm{mic}}}{\partial R_{\mathrm{pot}}}\left(1+\frac{B_{\mathrm{den}}}{R_{\text {den }}^{2}}\right) A^{1 / 3}\left(1+\bar{\epsilon}+r_{0} \frac{\partial \bar{\epsilon}}{\partial r_{0}}\right) \\
& +V_{a}\left(\frac{\partial E_{\mathrm{p}}^{\mathrm{mic}}}{\partial V_{\mathrm{p}}}-\frac{\partial E_{\mathrm{n}}^{\mathrm{mic}}}{\partial V_{\mathrm{n}}}\right) \frac{\partial \bar{\delta}}{\partial r_{0}}
\end{aligned}
$$

where

$$
\begin{aligned}
\frac{\partial \bar{\delta}}{\partial r_{0}} & =-\frac{3 c_{1}}{2 r_{0}} Z^{2} A^{-4 / 3}\left(4 Q A^{1 / 3}+9 J\right)^{-1} \\
\frac{\partial \bar{\epsilon}}{\partial r_{0}} & =-\frac{c_{1}}{K r_{0}} \frac{Z^{2}}{A^{4 / 3}}\left(\frac{3 L \bar{\delta}}{4 Q A^{1 / 3}+9 J}+1\right) .
\end{aligned}
$$

The derivative of $E^{\text {mic }}$ with respect to $a_{\text {pot }}$ is obtained separately. The derivatives of $E^{\text {mic }}$ with respect to both $C_{\mathrm{S}}$ and $r_{\text {mic }}$ are obtained without additional computation of single-particle energies. Using these expressions instead of using standard (but readily available) CONRAD "black box" numerical derivatives makes the analysis about 12 times faster. Related quantities can also be derived for the zero-vibration energy contribution, they are not reported here but are included in our calculations.

\section{Sensitivity analysis}

The sensitivity analysis shows that FRLDM parameters can be gathered into three groups of parameters according to their sensitivity weight. The group of the most sensitive parameters contains $a_{\mathrm{V}}, r_{0}, a_{\mathrm{S}}, \kappa_{\mathrm{V}}, \kappa_{\mathrm{S}}, a_{\mathrm{den}}, a$ and $r_{\mathrm{p}}$. The group of second-order sensitive parameters contains $V_{\mathrm{S}}$, $W, a_{\text {pot }}, l_{\mathrm{n}}, l_{\mathrm{p}}, c_{\mathrm{a}}, A_{\mathrm{den}}, C_{\mathrm{S}}, r_{\text {mic }}$ and $R$. Then parameters $a_{\mathrm{el}}, V_{\mathrm{a}}, k_{\mathrm{p}}, a_{2}, h, B_{\mathrm{den}}, J, Q, k_{\mathrm{n}}, K$ and $L$ have a limited impact on calculated masses. The present analysis focus on sensitivities on masses. In this case the most sensitive parameters have very simple $A-Z$ structure as can be seen for the most sensitive parameter $a_{\mathrm{V}}$ in Fig. 2.

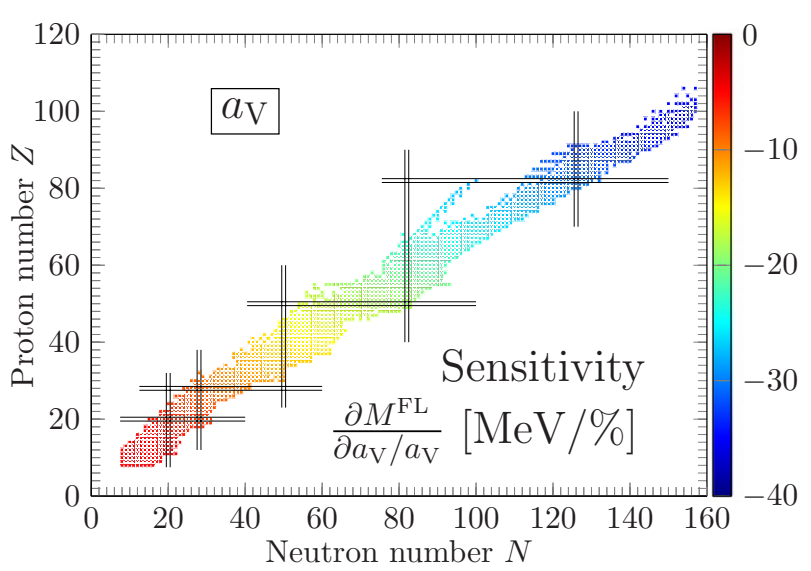

Figure 2. Mass sensitivity with respect to the most sensitive macroscopic parameter $a_{\mathrm{V}}$.

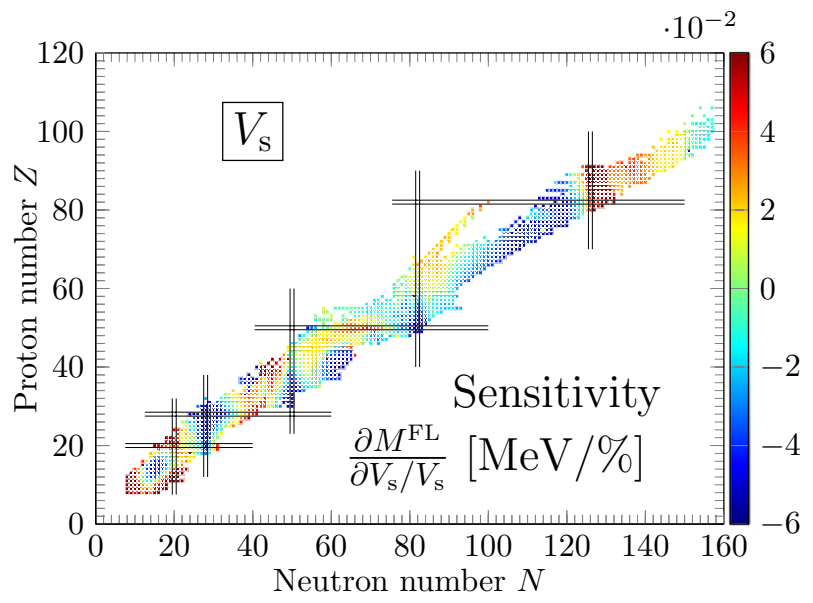

Figure 3. Mass sensitivity with respect to the most sensitive microscopic parameter $V_{\mathrm{S}}$.

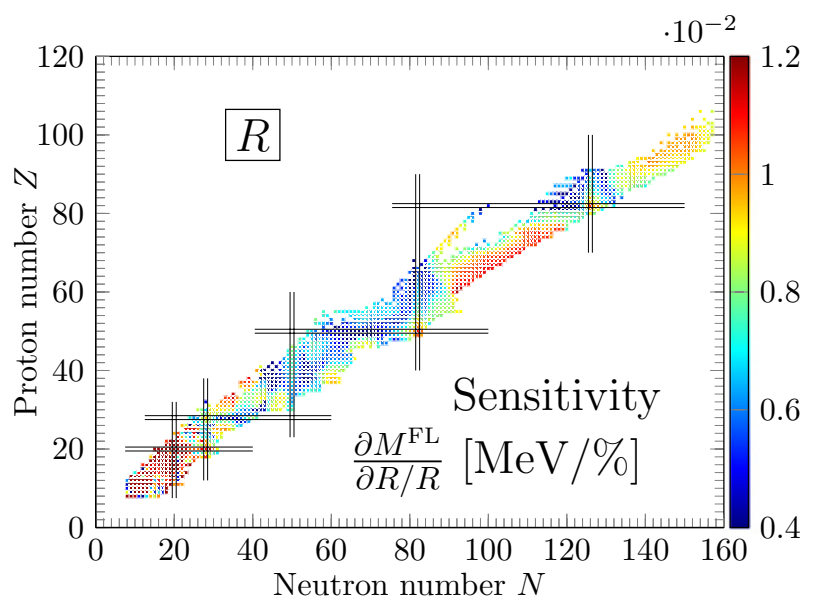

Figure 4. Mass sensitivity with respect to the zero-vibration energy parameter $R$.

The most sensitive parameters are also mainly shapeindependent and thus will have no impact on fission barrier. Therefore it is expected that second-order parameters for masses become more important for fission barrier heights. They also show more complex structures related to the nuclei internal microscopic configuration. An example of such finely structured sensitivities can be seen in Fig. 3 for the most sensitive microscopic parameter $V_{\mathrm{S}}$. 


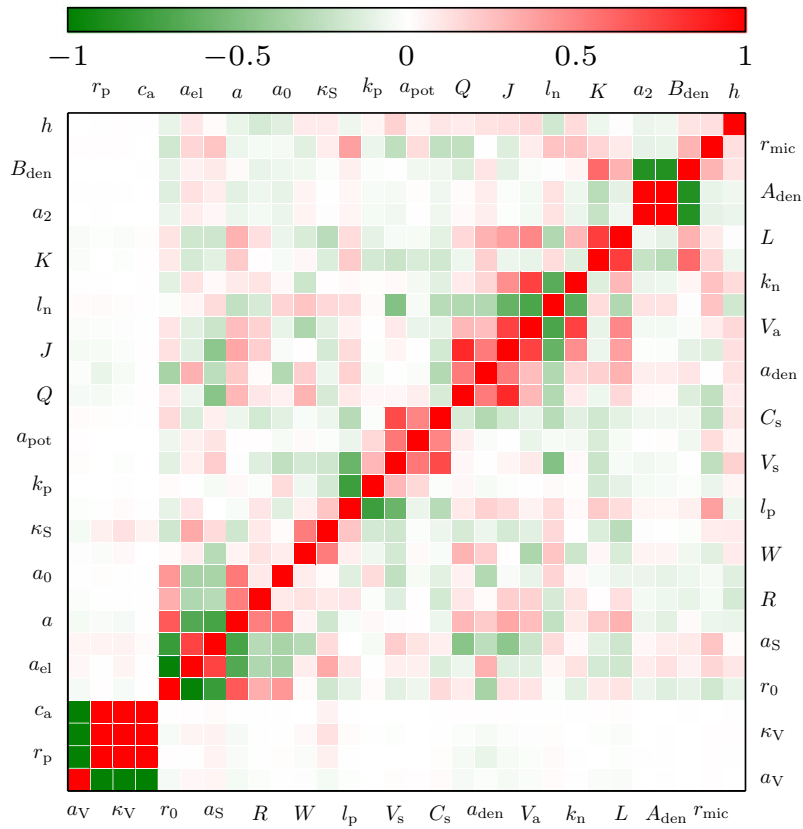

Figure 5. Full correlation matrix between FRLDM parameters. Parameter names are alternately placed on both sides.

Sensitivity with respect to the zero-vibration energy parameter $R$ is also shown in Fig. 4 as it presents similarities in the region $(Z=70, N=110$ ) with residual discrepancy against experimental data that are shown in the upper part of Fig. 1.

This is consistent with the fact that the most recent upgrade of the FRLDM [4], which mostly implies modifications of the zero-vibration energy calculation, reduces discrepancy with experimental data.

Finally, the CONRAD analysis methods can be used to provide correlation data between model parameters, see Fig. 5. The parameters correlation matrix shows that the most sensitive (macroscopic) shape-independent parameters are strongly correlated. Yet, less sensitive and shape-dependent parameters are not completely independent and thus must be properly considered while addressing uncertainty on fission barrier heights.

\section{References}

[1] D.L. Hill, J.A. Wheeler. Phys. Rev. 89, 1102 (1953)

[2] P. Möller, J.R. Nix, W.D. Myers, W.J. Swiatecki, Atomic Data and Nuclear Data Tables 59, 185, (1995)

[3] P. Archier, C. De Saint Jean, O. Litaize, G. Noguère, L. Berge, E. Privas, P. Tamagno, Nuclear Data Sheets 118, 488 (2014)

[4] P. Möller, A.J. Sierk, T. Ichikawa, H. Sagawa, Atomic Data and Nuclear Data Tables 109-110, 1 (2016) 